\title{
The analysis of a multiserver queue fed by a discrete autoregressive process of order $1^{1}$
}

\author{
Bong Dae Choi and Bara Kim \\ Department of Mathematics and Telecommunication Mathematics Research Center \\ Korea University, 1, Anam-dong, Sungbuk-ku, Seoul, 136-701, Korea \\ e-mail:bdchoi@semi.korea.ac.kr,bara@korea.ac.kr,
}

Gang Uk Hwang

Division of Applied Mathematics, Korea Advanced Institute of Science and Technology

373-1 Kusong-Dong, Yusong-Gu, Taejon 305-701, Korea

Jae-Kyoon Kim

Department of Electrical Engineering, Korea Advanced Institute of Science and Technology

373-1 Kusong-Dong, Yusong-Gu, Taejon 305-701, Korea

\begin{abstract}
We analyze a multiserver queue with a discrete autoregressive process of order 1 (DAR(1)) as an input. DAR(1) is a good mathematical model for VBR-coded teleconference traffic. Based on matrix analytic methods and the theory of Markov regenerative processes, we obtain the stationary distributions of the system size and the waiting time of an arbitrary packet. Numerical examples illustrate the quantitative effect of the stationary distribution and the autocorrelation function of input traffics on system performance.
\end{abstract}

keywords DAR(1), multiserver queue, Teleconference traffic, multiserver ATM multiplexer, matrix analytic methods, Markov regenerative theory, performance analysis

\footnotetext{
${ }^{1}$ This work was supported by Korea Research Foundation Grant (KRF-2001-042-D00009).
} 


\section{Introduction}

The discrete autoregressive process of order $1(\mathrm{DAR}(1))$ is known to be a good model for VBRcoded teleconference traffic (see [1]) and it is mathematically tractable for queueing models (see $[4,5])$. Moreover, it is described by a few parameters and it is easy to match the probability distribution and the decay rate of the autocorrelation function of $D A R(1)$ with those of the measured real traffic.

Recently, Hwang and Sohraby [4] and Hwang et al. [5] analyzed a discrete time single server queue with DAR(1) input. Hwang and Sohraby [4] obtained a closed form expression for the stationary probability generating function of the system size. Hwang et al. [5] obtained the waiting time distribution whose derivation was based on the analysis of the waiting time distribution of the discrete $G I / G / 1$ queue.

Kamoun and Ali [6] modeled an ATM multiplexer as a discrete time multiserver queueing system with on-off sources, and studied the transient and stationary distribution of the number of packets in the system.

In this paper, we consider the same multiserver ATM multiplexer as in [6], but with VBRcoded teleconference traffic input. Here we model the multiplexer as a discrete time multiserver queue with a DAR(1) input. We construct a Markov process at embedded epochs (see $\left\{\left(N_{k}, J_{k}\right)\right.$ : $k=0,1,2, \cdots\}$ in Section 3) whose matrix of one-step transition probabilities has $M / G / 1$ type, and calculate the stationary distribution of the constructed Markov process by matrix analytic methods. From the stationary distribution of this Markov process, we calculate the stationary distributions of the system size and the waiting time. Note that our approach is different from

that of Hwang and Sohraby [4] and Hwang et al. [5], and so our work is a nontrivial extension of 
$[4,5]$. Numerical examples support the intuitive fact that the distributions for the system size and the waiting time increase stochastically when either the decay rate of the autocorrelation function of input traffics or the variance of a batch arriving in a slot increases.

\section{Discrete autoregressive process of order 1 (DAR(1))}

Let $\{B(t): t=0,1,2 \cdots\}$ be a sequence of i.i.d. random variables. We assume that $B(t)$ takes nonnegative integer values and denote $b_{m}=P\{B(t)=m\}, m=0,1,2, \cdots$. Discrete Autoregressive Process of order 1 (abbreviated by $\operatorname{DAR}(1))\{X(t): t=0,1,2, \cdots\}$ is defined by the regression equation

$$
\begin{aligned}
& X(0)=B(0) \\
& X(t)=(1-\alpha(t)) X(t-1)+\alpha(t) B(t), \quad t=1,2,3, \cdots,
\end{aligned}
$$

where $\{\alpha(t): t=1,2,3, \cdots\}$ are i.i.d. Bernoulli random variables with $P\{\alpha(t)=0\}=\beta$ $(0 \leq \beta<1)$ and $P\{\alpha(t)=1\}=1-\beta$, and $\{\alpha(t): t=1,2,3 \cdots\}$ is assumed to be independent of $\{B(t): t=0,1,2 \cdots\}$. Note that $\operatorname{DAR}(1)$ is determined by parameter $\beta$ and distribution $\left\{b_{m}: m=0,1,2, \cdots\right\}$ of $B(t)$

The following properties are known for $\operatorname{DAR}(1)$ (see, for example, [5]).

- $\{X(t): t=0,1,2 \cdots\}$ is stationary.

- The probability distribution of $X(t)$ is the same one as the distribution of $B(t)$, i.e.,

$$
P\{X(t)=m\}=b_{m}, \quad m=0,1,2, \cdots .
$$


- The autocorrelation function $r(t)$ for $\{X(t)\}$ is given by

$$
r(t)=\frac{\operatorname{Cov}(X(0), X(t))}{\operatorname{Var}(X(0))}=\beta^{t}, \quad t=0,1,2, \cdots
$$

Note that the parameter $\beta$ is related to the decay rate of the autocorrelation function.

\section{Analysis of the $D A R(1) / D / c$ queue}

We consider the discrete time $D A R(1) / D / c$ queue, where the time is divided into slots of equal size and one slot is needed to serve a packet by a server. We assume that packet arrivals occur at the beginning of slots and departures occur at the end of slots. $\operatorname{ADAR}(1)\{X(t): t=0,1,2 \cdots\}$ represents packet arrivals so that $X(t)$ is the number of packets arriving at the beginning of the th slot.

We analyze the $D A R(1) / D / c$ queue. Let $N(t)$ be the number of packets in the system (we call it system size) immediately before arrivals at the beginning of the $t$ th slot. Then $\{(N(t), X(t))$ : $t=0,1,2, \cdots\}$ is a Markov process. Note that the Markov process $\{(N(t), X(t)): t=0,1,2, \cdots\}$ has $M / G / 1$ type [7]. But, it is not easy to calculate the stationary distribution of $\{(N(t), X(t))$ : $t=0,1,2, \cdots\}$ itself, because the number of phases is infinity. So, we find the stationary distribution of the Markov process $\{(N(t), X(t)): t=0,1,2, \cdots\}$ by introducing a new Markov process at the embedded epochs $\left\{\tau_{k}: k=0,1,2, \cdots\right\}$ defined below. Let $0=\tau_{0}<\tau_{1}<\tau_{2}<\cdots$ be the epochs defined by

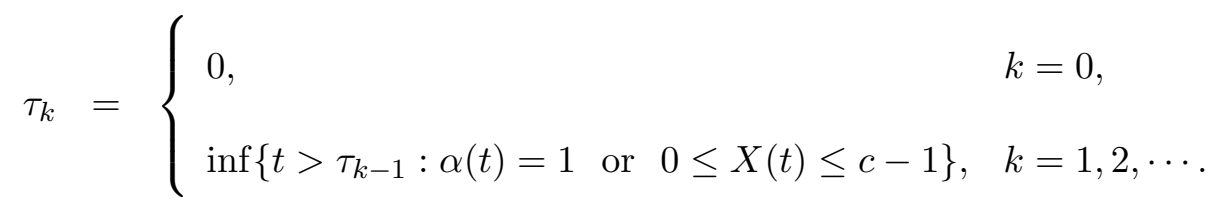


Let

$$
\begin{aligned}
N_{k} & =N\left(\tau_{k}\right), \quad k=0,1,2, \cdots, \\
J_{0} & =c, \\
J_{k} & =\left\{\begin{aligned}
X\left(\tau_{k}\right), & \text { if } \alpha\left(\tau_{k}\right)=0, \\
c, & \text { if } \alpha\left(\tau_{k}\right)=1,
\end{aligned}\right.
\end{aligned}
$$

Note that packet arrivals at and after $\tau_{k}$ are independent of the information prior to $\tau_{k}$ given $J_{k}$.

From this, it is observed that $\left\{\left(N_{k}, J_{k}\right): k=0,1,2, \cdots\right\}$ is a Markov process with state space $E=\{0,1,2, \cdots\} \times\{0, \cdots, c\}$, and that the Markov process $\left\{\left(N_{k}, J_{k}\right): k=0,1,2, \cdots\right\}$ has the following transition probabilities.

- For $n=0,1,2, \cdots$ and $i=0, \cdots, c-1$,

$$
(n, i) \rightarrow\left\{\begin{array}{cc}
(\max \{n-c+i, 0\}, i) & \text { with probability } \beta, \\
(\max \{n-c+i, 0\}, c) & \text { with probability } 1-\beta .
\end{array}\right.
$$

- For $n=0,1,2, \cdots$,

$$
(n, c) \rightarrow\left\{\begin{array}{llr}
(\max \{n-c+i, 0\}, i) & \text { with probability } b_{i} \beta, & 0 \leq i \leq c-1, \\
(n-c+i, c) & \text { with probability } b_{i}(1-\beta), & c-n+1 \leq i \leq c-1, \\
(0, c) & \text { with probability } \sum_{i=0}^{\min \{c-n, c-1\}} b_{i}(1-\beta)+g_{0} \delta_{n 0}, \\
(n+l, c) & \text { with probability } g_{l}, & l \geq 0, n+l>0,
\end{array}\right.
$$

where

$$
\begin{aligned}
\delta_{n 0} & =\left\{\begin{aligned}
1 & \text { if } n=0, \\
0 & \text { if } n \geq 1,
\end{aligned}\right. \\
g_{0} & =b_{c}, \\
g_{l} & =\sum_{i \mid l} b_{i+c}(1-\beta) \beta^{\frac{l}{i}-1}, \quad l=1,2, \cdots .
\end{aligned}
$$


Therefore, the Markov process $\left\{\left(N_{k}, J_{k}\right): k=0,1,2, \cdots\right\}$ has the $M / G / 1$ type structure (see [7]) of the one step transition probability matrix $P$ :

$$
P=\left[\begin{array}{cccccccccc}
B_{c} & A_{c+1} & A_{c+2} & \cdots & & & & & \\
B_{c-1} & A_{c} & A_{c+1} & A_{c+2} & \cdots & & & & \\
\vdots & \vdots & \vdots & \vdots & & & & & \\
B_{1} & A_{2} & A_{3} & A_{4} & A_{5} & \cdots & & & \\
A_{0} & A_{1} & A_{2} & A_{3} & A_{4} & A_{5} & \cdots & & \\
& A_{0} & A_{1} & A_{2} & A_{3} & A_{4} & A_{5} & \cdots & \\
& & & & & & & & \\
& & A_{0} & A_{1} & A_{2} & A_{3} & A_{4} & A_{5} & \cdots \\
& & & \ddots & \ddots & \ddots & \ddots & \ddots & \ddots
\end{array}\right],
$$

where

$$
\begin{aligned}
& \begin{array}{llllll}
0 & \cdots & i & \cdots & & c
\end{array} \\
& A_{i}=\begin{array}{c}
0 \\
\vdots \\
i \\
i \\
c
\end{array}\left[\begin{array}{ccccc}
0 & \cdots & 0 & \cdots & 0 \\
\vdots & & \vdots & & \vdots \\
0 & \cdots & \beta & \cdots & 1-\beta \\
\vdots & & \vdots & & \vdots \\
0 & \cdots & h_{i} \beta & \cdots & h_{i}(1-\beta)
\end{array}\right], \quad 0 \leq i \leq c-1, \\
& A_{i}=\begin{array}{c}
0 \\
0 \\
c
\end{array}\left[\begin{array}{ccc}
0 & \cdots & c \\
\vdots & & 0 \\
0 & \cdots & g_{i-c}
\end{array}\right], i \geq c \\
& B_{i}=\sum_{j=0}^{i} A_{j}, \quad 1 \leq i \leq c .
\end{aligned}
$$


We assume that the stability condition

$$
\lambda \triangleq E[X(t)]=\sum_{m=1}^{\infty} m b_{m}<c
$$

is satisfied. Then, by matrix analytic methods in [7], the limiting probabilities (hence the stationary probabilities) of $\left\{\left(N_{k}, J_{k}\right): k=0,1,2, \cdots\right\}$

$$
\pi_{n i}=\lim _{k \rightarrow \infty} P\left\{N_{k}=n, J_{k}=i\right\}, \quad n \geq 0,0 \leq i \leq c,
$$

are calculated as follows:

1. Set

$$
\tilde{A}_{n}=\left[\begin{array}{cccc}
A_{c n} & A_{c n+1} & \cdots & A_{c(n+1)-1} \\
A_{c n-1} & A_{c n} & \cdots & A_{c(n+1)-2} \\
\vdots & \vdots & \ddots & \vdots \\
A_{c(n-1)+1} & A_{c(n-1)+2} & \cdots & A_{c n}
\end{array}\right], n=0,1,2, \cdots
$$

where $A_{l}=O$ for $l<0$. Set

$$
\begin{gathered}
\tilde{B}_{0}=\left[\begin{array}{cccc}
B_{c} & A_{c+1} & \cdots & A_{2 c-1} \\
B_{c-1} & A_{c} & \cdots & A_{2 c-2} \\
\vdots & \vdots & \ddots & \vdots \\
B_{1} & A_{2} & \cdots & A_{c}
\end{array}\right], \\
\tilde{B}_{n}=\tilde{A}_{n+1}, \quad n=1,2, \cdots .
\end{gathered}
$$

2. Find the minimal nonnegative solution $G$ of the matrix equation

$$
G=\sum_{n=0}^{\infty} \tilde{A}_{n} G^{n}
$$


For example, $G$ is given by the iteration

$$
\begin{aligned}
G_{0} & =O \\
G_{l+1} & =\sum_{n=0}^{\infty} \tilde{A}_{n} G_{l}^{n}, \quad l=0,1,2, \cdots
\end{aligned}
$$

Set

$$
K=\sum_{n=0}^{\infty} \tilde{B}_{n} G^{n} .
$$

3. Find a positive row vector $\kappa$ satisfying

$$
\kappa K=\kappa .
$$

4. Set

$$
\begin{aligned}
x_{0} & =\kappa \\
x_{n} & =\left(x_{0} \sum_{i=0}^{\infty} \tilde{B}_{n+i} G^{i}+\sum_{l=1}^{n-1} x_{l} \sum_{i=0}^{\infty} \tilde{A}_{n-l+i+1} G^{i}\right)\left(I-\sum_{i=0}^{\infty} \tilde{A}_{i+1} G^{i}\right)^{-1}, n=1,2, \cdots
\end{aligned}
$$

5. Finally,

$$
\left(\left(\pi_{n c, 0}, \cdots, \pi_{n c, c}\right), \cdots,\left(\pi_{(n+1) c-1,0}, \cdots, \pi_{(n+1) c-1, c}\right)\right)=C x_{n}, \quad n=0,1,2, \cdots,
$$

where $C^{-1}=\sum_{n=0}^{\infty} x_{n} e$ and $e$ is the $c(c+1)$-dimensional column vector whose components are all ones.

Now we find the stationary distribution of the Markov process $\{(N(t), X(t)): t=0,1,2, \cdots\}$. Observe that $\left\{\left(\left(N_{k}, J_{k}\right), \tau_{k}\right): k=0,1,2, \cdots\right\}$ is a Markov renewal sequence (see p. 479 in [8]) and that $\left\{\left(N\left(t+\tau_{k}\right), X\left(t+\tau_{k}\right)\right): t=0,1,2, \cdots\right\}$ given $\left\{(N(u), X(u)), 0 \leq u<\tau_{k},\left(N_{k}, J_{k}\right)=(n, i)\right\}$ 
is stochastically equivalent to $\left\{(N(t), X(t): t=0,1,2, \cdots\}\right.$ given $\left\{\left(N_{0}, J_{0}\right)=(n, i)\right\}$. Hence $\{(N(t), X(t)): t=0,1,2, \cdots\}$ is a discrete time Markov regenerative process (see Definition 3 in Appendix) with the Markov renewal sequence $\left\{\left(\left(N_{k}, J_{k}\right), \tau_{k}\right): k=0,1,2, \cdots\right\}$.

By Theorem 4 in Appendix, the limiting probabilities (hence the stationary probabilities) $p_{n j}=\lim _{t \rightarrow \infty} P\{(N(t), X(t))=(n, j)\}, n, j=0,1,2, \cdots$, of $\{(N(t), X(t)): t=0,1,2, \cdots\}$ are given by

$$
p_{n j}=\frac{\sum_{l=0}^{\infty} \sum_{i=0}^{c} \pi_{l i} E\left[\sum_{t=\tau_{k}}^{\tau_{k+1}-1} 1_{\{(N(t), X(t))=(n, j)\}} \mid\left(N_{k}, J_{k}\right)=(l, i)\right]}{\sum_{l=0}^{\infty} \sum_{i=0}^{c} \pi_{l i} E\left[\tau_{k+1}-\tau_{k} \mid\left(N_{k}, J_{k}\right)=(l, i)\right]} .
$$

Observe that

$$
\begin{aligned}
& E\left[\sum_{t=\tau_{k}}^{\tau_{k+1}-1} 1_{\{(N(t), X(t))=(n, j)\}} \mid\left(N_{k}, J_{k}\right)=(l, i)\right] \\
& = \begin{cases}1 & \text { if } i=j, 0 \leq i \leq c-1 \text { and } n=l, \\
b_{j} & \text { if } i=c, 0 \leq j \leq c-1 \text { and } n=l, \\
\frac{b_{c}}{1-\beta} & \text { if } i=c, j=c \text { and } n=l, \\
b_{j} \beta^{\frac{n-l}{j-c}} & \text { if } i=c, j>c, n \geq l \text { and } j-c \text { divides } n-l, \\
0 & \text { otherwise. }\end{cases}
\end{aligned}
$$

Therefore the numerator of the right hand side of (3) is

$$
\begin{cases}\pi_{n j}+\pi_{n c} b_{j}, & 0 \leq j \leq c-1, \\ \frac{\pi_{n c} b_{c}}{1-\beta}, & j=c, \\ \sum_{i=0}^{\left\lfloor\frac{n}{j-c}\right\rfloor} \pi_{n-i(j-c), c} b_{j} \beta^{i}, & j \geq c+1 .\end{cases}
$$

Observe that

$$
E\left[\tau_{k+1}-\tau_{k} \mid\left(N_{k}, J_{k}\right)=(l, i)\right]= \begin{cases}1 & \text { if } 0 \leq i \leq c-1, \\ \sum_{s=0}^{c-1} b_{s}+\sum_{s=c}^{\infty} b_{s} \frac{1}{1-\beta} & \text { if } i=c .\end{cases}
$$


Therefore the denominator of the right hand side of (3) is

$$
\sum_{l=0}^{\infty} \sum_{i=0}^{c-1} \pi_{l i}+\sum_{l=0}^{\infty} \pi_{l c}\left(\sum_{s=0}^{c-1} b_{s}+\sum_{s=c}^{\infty} b_{s} \frac{1}{1-\beta}\right) .
$$

Observe that $\left(\sum_{l=0}^{\infty} \pi_{l 0}, \cdots, \sum_{l=0}^{\infty} \pi_{l c}\right)$ is the stationary probability vector of the Markov process $\left\{J_{k}: k=0,1,2, \cdots\right\}$ whose transition probability matrix is

$$
\left(P\left(J_{k+1}=j \mid J_{k}=i\right)\right)_{0 \leq i, j \leq c}=\left[\begin{array}{ccccc}
\beta & 0 & \cdots & 0 & 1-\beta \\
0 & \beta & \cdots & 0 & 1-\beta \\
\vdots & \vdots & \ddots & \vdots & \vdots \\
0 & 0 & \cdots & \beta & 1-\beta \\
\beta b_{0} & \beta b_{1} & \cdots & \beta b_{c-1} & 1-\beta \sum_{s=0}^{c-1} b_{s}
\end{array}\right] .
$$

By solving the balance equations for the stationary distribution of the Markov process $\left\{J_{k}: k=\right.$ $0,1,2, \cdots\}$, we obtain

$$
\sum_{l=0}^{\infty} \pi_{l i}= \begin{cases}\frac{\beta b_{i}}{1-\beta \sum_{s=c}^{\infty} b_{s}}, & 0 \leq i \leq c-1, \\ \frac{1-\beta}{1-\beta \sum_{s=c}^{\infty} b_{s}}, & i=c .\end{cases}
$$

By substituting (5) into (4), we obtain the denominator of the right hand side of (3) as $\left(1-\beta \sum_{s=c}^{\infty} b_{s}\right)^{-1}$.

Thus, we have the following theorem.

Theorem 1 The limiting probabilities (hence the stationary probabilities)

$p_{n j}=\lim _{t \rightarrow \infty} P\{(N(t), X(t))=(n, j)\}, n, j=0,1,2, \cdots$, of $\{(N(t), X(t)): t=0,1,2, \cdots\}$ are given by

$$
p_{n j}= \begin{cases}\mu^{-1}\left(\pi_{n j}+\pi_{n c} b_{j}\right), & 0 \leq j \leq c-1, \\ \mu^{-1} \frac{\pi_{n c} b_{c}}{(1-\beta)}, & j=c, \\ \mu^{-1} \sum_{i=0}^{\left\lfloor\frac{n}{j-c}\right\rfloor} \pi_{n-i(j-c), c} b_{j} \beta^{i}, & j \geq c+1,\end{cases}
$$


where $\mu^{-1}=1-\beta \sum_{s=c}^{\infty} b_{s}$.

Now we find the stationary distribution of the waiting time of a packet. Let $W$ denote the waiting time of an arbitrary packet at steady state. Then for $w=0,1,2, \cdots$,

$$
\begin{aligned}
& P(W=w) \\
& =\frac{\text { Mean number of arrivals in a slot at steady state whose waiting time is } w}{\text { Mean number of arrivals in a slot }} .
\end{aligned}
$$

Suppose that there are $n$ packets immediately before arrivals at the beginning of the $t$ th slot and that the number of packet arrivals is $j$ at the beginning of the $t$ th slot, i.e., $N(t)=n$ and $X(t)=j$. Then the number of packets whose waiting time is $w$ among the ones who arrive at the beginning of the $t$ th slot is

$$
\begin{cases}\min \{c(w+1)-n, j\}, & c w<n<c(w+1) \\ \min \{n+j-c w, c\}, & n \leq c w<n+j \\ 0, & \text { otherwise. }\end{cases}
$$

Therefore the mean number of arrivals in a slot at steady state whose waiting time is $w$ is

$$
\sum_{n=0}^{c w} \sum_{j=c w-n+1}^{\infty} p_{n j} \min \{n+j-c w, c\}+\sum_{n=c w+1}^{c(w+1)-1} \sum_{j=1}^{\infty} p_{n j} \min \{c(w+1)-n, j\}
$$

Since the mean number of arrivals in a slot is $\lambda$, the following theorem is obtained from (6).

Theorem 2 The distribution of the waiting time $W$ of an arbitrary packet is given by

$$
\begin{aligned}
& P(W=w) \\
& =\frac{1}{\lambda}\left(\sum_{n=0}^{c w} \sum_{j=c w-n+1}^{\infty} p_{n j} \min \{n+j-c w, c\}+\sum_{n=c w+1}^{c(w+1)-1} \sum_{j=1}^{\infty} p_{n j} \min \{c(w+1)-n, j\}\right), \\
& w=0,1,2, \cdots .
\end{aligned}
$$




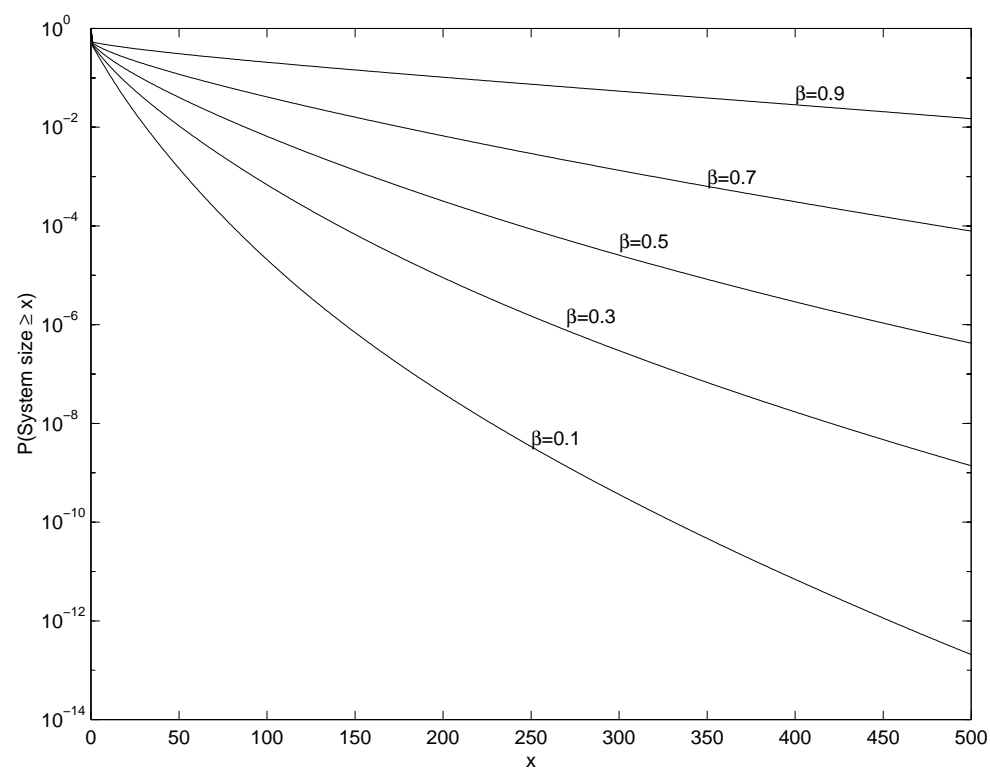

Figure 1: Complementary distribution function of the stationary system size, when $m=1$

\section{Numerical Examples}

It is shown that the stationary distribution of VBR-coded video teleconference traffics is a negative binomial distribution $[1,2,3]$. For numerical examples, we choose negative binomial distributions as the stationary distribution of $\operatorname{DAR}(1)$, i.e.,

$$
b_{k}=\left(\begin{array}{c}
k+m-1 \\
m-1
\end{array}\right) q^{m}(1-q)^{k}, \quad k=0,1,2, \cdots
$$

Here mean and variance of the negative binomial distribution are $m \times \frac{1-q}{q}$ and $m \times \frac{1-q}{q^{2}}$, respectively. To get a fixed mean number 3.5 of the negative binomial distribution for various values of $m$, let $m \times \frac{1-q}{q}=3.5$ so that $q=\frac{2 m}{2 m+7}$. Then the variance is $m \times \frac{1-q}{q^{2}}=3.5+\frac{49}{4 m}$. Let the number of servers is $c=5$. Note that the offered load is $\frac{\lambda}{c}=0.7$.

Figure 1 and Figure 2 display the complementary distribution functions of the stationary 


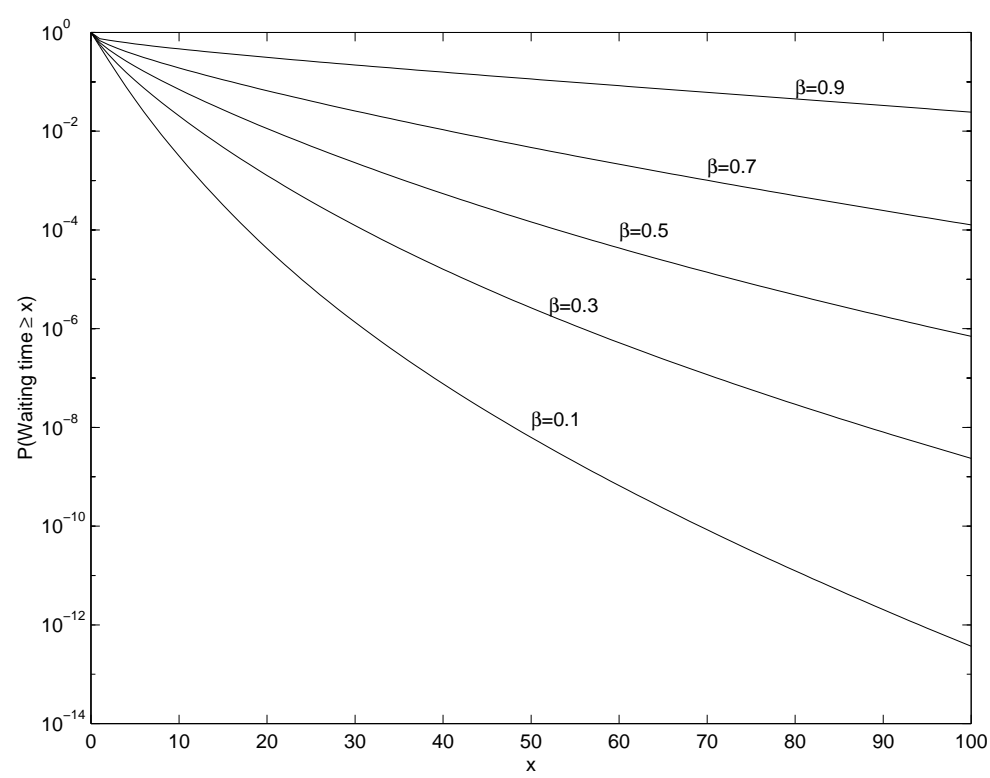

Figure 2: Complementary distribution function of the waiting time of an arbitrary packet, when $m=1$.

system size and the waiting time of an arbitrary packet, respectively, when $m=1$ and $\beta=0.1$, 0.3, 0.5, 0.7 and 0.9. From (1), the parameter $\beta$ gives information on the strength of correlation of the input process. The larger the parameter $\beta$ is, the slower the decay of the autocorrelation of the input process is. So it is expected that stationary system size and waiting time for the case of large $\beta$ (for example, $\beta=0.9$ ) are stochastically larger than those for the case of small $\beta$ (for example, $\beta=0.1$ ). Figure 1 and Figure 2 support this intuitive fact.

Figure 3 and Figure 4 display the complementary distribution functions of the stationary system size and waiting time of an arbitrary packet, respectively, when $\beta=0.3$ and $m=1,2,3$ and 4. It is intuitive that stationary system size and waiting time for the case that the stationary distribution of the input process has a large variance (for example, $m=1$ ) are stochastically larger than those for the case that the stationary distribution of the input process has a small 


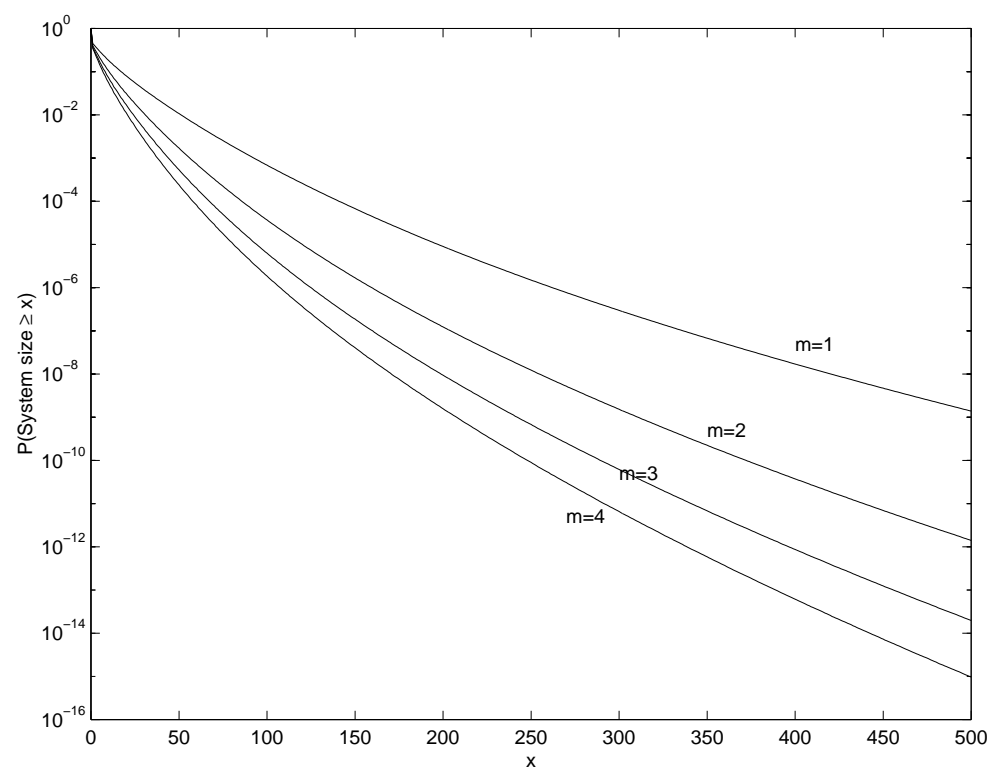

Figure 3: Complementary distribution function of the stationary system size, when $\beta=0.3$.

variance (for example, $m=4$ ). Figure 3 and Figure 4 support this intuitive fact.

\section{Conclusions}

In the paper, we analyze a multiserver queue fed by a $\operatorname{DAR}(1)$ input which is a good mathematical model for a multiserver ATM multiplexer with VBR-coded teleconferece traffic input. Based on matrix analytic methods and the theory of Markov regenerative processes, we obtain the stationary distributions of the system size and the waiting time of an arbitrary packet. Numerical examples show that the distributions for the system size and the waiting time increase stochastically when either the decay rate $\beta$ of the autocorrelation function of input traffics or the variance of a batch arriving in a slot increases. 


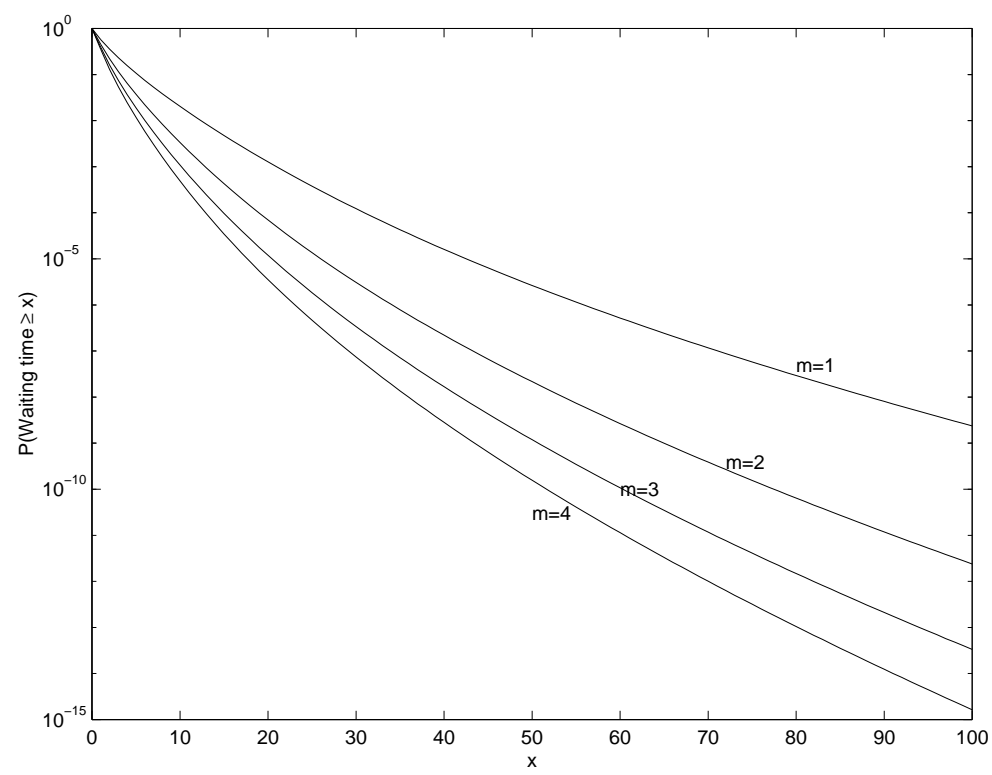

Figure 4: Complementary distribution function of the waiting time of an arbitrary packet, when $\beta=0.3$.

\section{APPENDIX}

We deal with the discrete time version of Markov regenerative processes. For the (continuous time) Markov regenerative processes, see for example p.525-532 in [8].

Definition $3 A$ discrete time process $\{Z(t): t=0,2,3, \cdots\}$ is a discrete time Markov regenerative process if there exists a Markov renewal sequence $\left\{\left(Y_{k}, S_{k}\right): k=0,1,2, \cdots\right\}$ such that all conditional finite dimensional distributions of $\left\{Z\left(t+S_{k}\right): t=0,1,2, \cdots\right\}$ given $\{Z(u), 0 \leq u<$ $\left.S_{k}, Y_{k}=i\right\}$ are the same as those of $\{Z(t): t=0,1,2, \cdots\}$ given $\left\{Y_{0}=i\right\}$.

The following is a limit theorem for discrete time Markov regenerative processes, which is proved by similar procedure as its continuous counterpart. See p.527 in [8] for the continuous counterpart. 
Theorem 4 Let $\{Z(t): t=0,1,2 \cdots\}$ be a discrete time Markov regenerative process on the countable state space $\mathcal{S}$ with Markov renewal sequence $\left\{\left(Y_{k}, S_{k}\right): k=0,1,2, \cdots\right\}$. Let $\mathcal{E}$ be the countable state space of the Markov process $\left\{Y_{k}: k=0,1,2, \cdots\right\}$ and for $i \in \mathcal{E}$ and $j \in \mathcal{S}$,

$$
\begin{aligned}
\mu_{i} & =E\left[S_{1} \mid Y_{0}=i\right] \\
\alpha_{i j} & =E\left[\sum_{t=0}^{S_{1}-1} 1_{\{Z(t)=j\}} \mid Y_{0}=i\right]
\end{aligned}
$$

Suppose that the discrete time semi-Markov process $\{Y(t): t=0,1,2, \cdots\}$ defined by $Y(t)=Y_{k}$

for $S_{k} \leq t<S_{k+1}$ is irreducible, aperiodic and positive recurrent. Then for $k \in \mathcal{E}$ and $j \in \mathcal{S}$,

$$
\lim _{t \rightarrow \infty} P\left\{Z(t)=j \mid Y_{0}=k\right\}=\frac{\sum_{i \in \mathcal{E}} \pi_{i} \alpha_{i j}}{\sum_{i \in \mathcal{E}} \pi_{i} \mu_{i}},
$$

where $\pi=\left(\pi_{i}\right)_{i \in \mathcal{E}}$ is a stationary measure of the Markov process $\left\{Y_{k}: k=0,1,2, \cdots\right\}$.

\section{References}

[1] A. Elwalid, D. Heyman, T.V. Laksman, D. Mitra and A. Weiss, Fundamental Bounds and Approximations for ATM Multiplexers with Applications to Video Teleconferencing, IEEE Journal of Selected Areas in Communications, Vol. 13, No. 6, pp. 1004-1016, 1995.

[2] D. P. Heyman, A Tabatabai and T. V. Lakshman, Statistical analysis and simulation study of a video teleconference traffic in ATM networks, IEEE Trans. Circuits, Syst., Video Technol., Vol. 2, No. 1, pp. 49-59, Mar. 1992.

[3] D. P. Heyman, T. V. Lakshman, A Tabatabai and H. Heeke, Modeling teleconference traffic from VBR video coders, Proc. ICC 1994, pp. 1744-1748, 1994. 
[4] G.U. Hwang and K. Sohraby, On the exact analysis of a discrete-time queueing system with autoregressive inputs, Queueing Systems, Vol. 43, No. 1-2, pp.29-41, 2003.

[5] G.U. Hwang, B. D. Choi and J.-K. Kim, The waiting time analysis of a discrete time queue with arrivals as an autoregressive process of order 1, Journal of Applied Probability, Vol. 39, No. 3, pp.619-629, 2002.

[6] F. Kamoun and M. M. Ali, A new theoretical approach for the transient and steady-state analysis of multiserver ATM multiplexers with correlated arrivals, Proc. ICC 1995, Vol. 2, pp. 1127-1131, 1995.

[7] M.F. Neuts, Structured stochastic matrices of the $M / G / 1$ type and their applications, Dekker, New York, 1989.

[8] V.G. Kulkarni, Modeling and analysis of stochastic systems, Chapman \& Hall, 1995. 